\title{
Déshydratation-imprégnation par immersion de rondelles de mangue (Mangifera indica) : influence de la température et de la concentration de la solution sur les cinétiques de certains éléments constitutifs du fruit
}

\author{
Yvette Jiokap Nono ${ }^{a \star}$, Guy Bertin Nuadje ${ }^{a}$, Anne-Lucie Raoult-Wack ${ }^{\text {b }}$, François Giroux ${ }^{c}$
}

\author{
a Département de Génie \\ des procédés et d'ingénierie, \\ École nationale supérieure \\ des Sciences agro-industrielles \\ (ENSAI), \\ Université de Ngaoundéré, \\ BP 455, \\ Ngaoundéré, Cameroun \\ b Programme agro-alimentaire, \\ Cirad-amis, \\ 73 rue J.-F. Breton, \\ TA 40/16, 34398 Montpellier \\ Cedex 01, France \\ ${ }^{c}$ Ensia-Siarc, \\ 1101, Avenue d'Agropolis, \\ BP 5098, \\ 34033 Montpellier Cedex 01, \\ France
}

${ }^{*}$ Correspondance et tirés à part

Reçu le 7 juillet 2000

Accepté le 30 novembre 2000

Fruits, 2001, vol. 56, p. 169-177 (C) $2001 \mathrm{Cirad} /$ EDP Sciences All rights reserved

Resumen Español, p. 177

\section{Dehydrating-impregnation soaking (DIS) process of mango pieces (Mangifera indica): solution temperature and concentration effects on the kinetics of certain fruit components.}

Abstract - Introduction. Matter transfers intervening during the process of DIS influence the final composition of the product. These transfers primarily depend on the form and the size of the soaked products, the temperature, the concentration and the nature of the soaking solution. To obtain a good control of these parameters and to obtain a product of quality after processing, the kinetics of certain components of mangos (Mangifera indica) was followed during a process by DIS. Materials and methods. Slices of a Cameroon mango local variety, $9 \mathrm{~mm}$ in thickness and $31 \mathrm{~mm}$ in diameter, were soaked in a 50, 60 or $70{ }^{\circ}$ Brix sucrose solution, kept at a temperature stabilized at 30,50 or $60^{\circ} \mathrm{C}$. For the mango slices, the parameters followed during the process were the water loss, the weight reduction, the solute gain and the Brix value, while, for the solution, it was the vitamin $\mathrm{C}$ concentration, the $\mathrm{pH}$ and the conductivity which were measured. Results and discussion. The water loss and the final Brix degree of the samples increased with the temperature and the sucrose solution concentration. Beyond $50{ }^{\circ} \mathrm{C}$, the increase in the temperature did not significantly influence the weight reduction which, for a given temperature, increased with the sucrose solution concentration. The solute gain decreased with the increase in the solution concentration. Lastly, for the operating conditions tested, the losses in vitamin $\mathrm{C}$ increased with the temperature and decreased with the increase in the sucrose solution concentration.

Cameroon / Mangifera indica / conservation (storage) / dipping / temperature / sucrose / concentrating / osmotic dehydration / diffusion / ascorbic acid / solutes / moisture content / carbohydrate content / pH / electrical conductivity

\section{Déshydratation-imprégnation par immersion (DII) de rondelles de mangue \\ (Mangifera indica) : influence de la température et de la concentration} de la solution sur les cinétiques de certains éléments constitutifs du fruit.

Résumé - Introduction. Les transferts de matière intervenant au cours du procédé de DII influencent la composition finale du produit. Ces transferts dépendent essentiellement de la forme et de la taille des produits immergés, ainsi que de la température, la concentration et la nature de la solution d'immersion. Afin de parvenir à une bonne maîtrise de ces paramètres et d'obtenir, après traitement, un produit de qualité, les cinétiques de certains éléments constitutifs de mangues (Mangifera indica) ont été suivies au cours d'un traitement par DII. Matériel et méthodes. Des morceaux de mangues d'une variété camerounaise locale, de $9 \mathrm{~mm}$ d'épaisseur et de $31 \mathrm{~mm}$ de diamètre, ont été immergés dans une solution de saccharose à 50,60 ou $70^{\circ}$ Brix, maintenue à une température stabilisée à 30,50 ou $60^{\circ} \mathrm{C}$. Pour les échantillons, les paramètres suivis au cours du temps ont été la perte d'eau, la réduction de poids, le gain en soluté et le degré Brix, tandis que, pour la solution, ce sont la concentration en vitamine $\mathrm{C}$, le $\mathrm{pH}$ et la conductivité qui ont été mesurés. Résultats et discussion. La perte d'eau et le degré Brix final des produits ont augmenté avec la température et la concentration de la solution. Au-delà de $50^{\circ} \mathrm{C}$, l'augmentation de la température n'a pas influencé significativement la réduction de poids qui, pour une température donnée, a augmenté avec la concentration de la solution d'immersion. Le gain en solutés a diminué avec l'augmentation de la concentration. Enfin, pour les conditions opératoires testées, les pertes en vitamine $C$ ont augmenté avec la température et diminué avec l'augmentation de la concentration de la solution de saccharose.

Cameroun / Mangifera indica / conservation (stockage) / immersion / température / saccharose / concentration / déshydratation osmotique / diffusion / acide ascorbique / soluté / teneur en eau / teneur en glucides / pH / conductivité électrique 


\section{Introduction}

Le manguier (Mangifera indica) est un arbre de la famille des anacardiacées qui pousse essentiellement en région tropicale et subtropicale. Son fruit de forme ovoïde, elliptique ou oblongue a une pulpe comestible constituée essentiellement de 80 à $90 \%$ d'eau et de 10 à $16 \%$ de glucides [1, 2]. Des protéines, lipides, acides organiques, sels minéraux et vitamines entrent aussi dans sa composition, mais en plus faibles quantités $[2,3]$. La pulpe de la mangue représente environ 60 à $75 \%$ de la masse totale du fruit [ 1 , 2]. Selon les variétés, les fruits ont, à maturité, des dimensions (longueur de 8 à $30 \mathrm{~cm}$ ) et des couleurs (vert au jaune, virant parfois au rouge [1]) variables.

Les mangues sont des fruits tropicaux très appréciés, surtout par les populations africaines qui les consomment en dessert, de préférence à l'état frais mais aussi en confitures, jus et mangues confites, séchées ou en conserves. Certains prétraitements peuvent être effectués pour améliorer la qualité du produit fini. Parmi eux, la technique de déshydratation-imprégnation par immersion, notée DII, occupe une place de choix. Pour être stables, les produits ainsi obtenus doivent être séchés, pasteurisés, congelés, ou encore incorporés dans d'autres préparations.

La DII consiste à mettre en contact des produits entiers ou découpés avec des solutions concentrées sucrées et/ ou salées, choisies en fonction des caractéristiques organoleptiques du produit traité. Les transferts de matière qui ont lieu au cours de ce procédé influencent la composition finale du produit. Ces transferts dépendent essentiellement de certaines variables telles que la forme et la taille des produits immergés, la température, la concentration et la nature de la solution d'immersion. Lors du traitement par DII de mangues, si la perte en eau est souhaitée, il n'en est pas de même pour les constituants nobles du fruit tels que les vitamines et les sels minéraux. De même, le transfert de solutés de la solution vers le produit doit être bien contrôlé. Seule une bonne maîtrise de ces paramètres permettra de proposer aux consommateurs un produit dont les caractéristiques nutritionnelles et organoleptiques se rapprocheront le plus de celles du produit naturel.

De nombreux travaux étudiant le comportement des mangues pendant la DII ont été publiés [4-8]. Toutefois, ils ont porté sur différentes variétés de mangues traitées dans des conditions expérimentales non comparables et ils sont donc difficilement extrapolables. L'objectif des recherches présentées a été d'étudier l'influence de la température et de la concentration de la solution d'immersion sur les principaux paramètres du procédé de DII de mangues d'une variété locale camerounaise non encore explorée en DII.

\section{Matériel et méthodes}

Les mangues traitées dans les expérimentations qui vont suivre appartiennent à une variété locale greffée, exploitée dans un verger de la région de Ngaoundéré, province de l'Adamaoua (Cameroun). Après leur récolte, les fruits de $22 \mathrm{~cm}$ de longueur en moyenne ont été conservés pendant une semaine à la température ambiante jusqu'à maturité adéquate. Avant d'être parés, ils ont alors été lavés, trempés pendant $30 \mathrm{~min}$ dans une solution aqueuse d'hypochlorite de sodium à $0,08 \%$ de chlore actif, rincés à l'eau courante, puis égouttés. Les fruits parés ont été découpés en disques de $9 \mathrm{~mm}$ d'épaisseur et $31 \mathrm{~mm}$ de diamètre. Leurs caractéristiques ont été évaluées en début d'expérimentation (tableau I).

Les disques de mangues ont été enveloppés dans du papier d'aluminium afin de les protéger de la lumière. Les déterminations de matière sèche et de cendres des échantillons ont été effectuées selon les normes AOAC [9]. Le dosage de la vitamine $\mathrm{C}$ a été fait par la méthode au 2,6-dichloroindophénol [10]. Pendant le traitement de DII, les fruits ont été immergés dans des solutions concentrées de saccharose. Le rapport massique moyen [fruits/solution] utilisé pour toutes les analyses de cinétique a été fixé à $[1 / 6,5]$. Le degré Brix des solutions de saccharose et celui des jus extraits des fruits après broyage ont été mesurés au réfractomètre. La solution d'immersion 


\begin{tabular}{|c|c|c|c|}
\hline $\begin{array}{l}{ }^{\circ} \text { Brix } \\
\left(\mathrm{g} \times 100 \mathrm{~g}^{-1} \text { de jus }\right)\end{array}$ & $\begin{array}{c}\text { Teneur en eau } \\
\left(\mathrm{g} \times 100 \mathrm{~g}^{-1} \mathrm{mf}\right)\end{array}$ & $\begin{array}{c}\text { Cendres } \\
\left(\mathrm{g} \times 100 \mathrm{~g}^{-1} \mathrm{~ms}\right)\end{array}$ & $\begin{array}{l}\text { Teneur en vitamine } \mathrm{C} \\
\left(\mathrm{mg} \times 100 \mathrm{~g}^{-1} \mathrm{mf}\right)\end{array}$ \\
\hline 13,1 & 86,1 & 4,5 & 41,7 \\
\hline
\end{tabular}

utilisée pendant le procédé a été préparée la veille de l'expérimentation avec de l'eau distillée afin de permettre une bonne dissolution du saccharose. Chaque expérience a été répétée deux fois. Les écarts entre les résultats de ces répétitions ont été inférieurs à $5 \%$.

L'étude cinétique des échanges intervenant pendant le procédé de DII a été conduite dans un bain thermostaté. Pour cela, dix pots en verre d'une contenance de $800 \mathrm{~mL}$ ont été utilisés pour étudier une gamme de dix temps expérimentaux fixés à $0,15,30,45,60,100,150,200,250$ et 300 min. Le couvercle de chaque pot a été équipé d'une grille permettant de faciliter l'immersion des disques de mangues pendant la déshydratation. Après stabilisation de la température dans les pots remplis de la solution concentrée sucrée, mis au bainmarie, les échantillons ont pu être introduits. Les pots destinés aux plus longues durées d'expérimentation ont été garnis les premiers. Un soin particulier a été apporté afin que les rondelles soient complètement immergées. Les bocaux ont été légèrement agités à la main toutes les $5 \mathrm{~min}$. À la fin des temps d'expérimentation impartis pour chacun des dix bocaux, la conductivité et le $\mathrm{pH}$ des solutions ont été mesurés. La gamme des températures a varié de $30{ }^{\circ} \mathrm{C}$ à $60^{\circ} \mathrm{C}$ et celle de la concentration des solutions de $50^{\circ}$ Brix à $70^{\circ}$ Brix.

Les transferts de matières ont été suivis par divers paramètres [11], définis à partir des paramètres définis dans le tableau II :

- La perte d'eau à l'instant $t$ :

$$
\left.\mathrm{PE}(\mathrm{t})=\mathrm{H}\left(\mathrm{t}_{0}\right)-\left\{\mathrm{M}_{\mathrm{p}}(\mathrm{t}) / \mathrm{M}_{\mathrm{p}}\left(\mathrm{t}_{0}\right)\right] \times \mathrm{H}(\mathrm{t})\right\} \text {. }
$$

- La réduction de poids à l'instant $t$ :

$$
R P(t)=\left[M_{p}\left(t_{0}\right)-M_{p}(t)\right] / M_{p}\left(t_{0}\right) .
$$

- Le gain en soluté à l'instant $t$ :

$$
\mathrm{GS}(\mathrm{t})=\mathrm{PE}(\mathrm{t})-\mathrm{RP}(\mathrm{t}) \text {. }
$$

- La masse de sucre dans l'échantillon à l'instant $t$ :

$$
\begin{gathered}
S(t)=\left\{B\left(t_{0}\right) \times M_{p}\left(t_{0}\right) \times H\left(t_{0}\right)\right] / \\
\left.\left[1-B\left(t_{0}\right)\right]\right\}+\left[G S(t) \times M_{p}\left(t_{0}\right)\right] .
\end{gathered}
$$

\begin{tabular}{|c|c|c|}
\hline Notation & Signification & Unité \\
\hline $\operatorname{PE}(t)$ & Perte d'eau à l'instant $t$, relativement à la masse initiale du produit ; $0 \leq \mathrm{PE}<1$ & $\mathrm{~g} / \mathrm{g}$ produit initial \\
\hline $\mathrm{S}(t)$ & Masse de sucre dans l'échantillon à l'instant $t$ & g \\
\hline $\mathrm{GS}(t)$ & Gain en soluté à l'instant $t$, relativement à la masse initiale du produit ; $0 \leq \mathrm{GS}<1$ & $\mathrm{~g} / \mathrm{g}$ produit initial \\
\hline $\mathrm{RP}(t)$ & Réduction de poids à l'instant $t$, relativement à la masse initiale du produit ; $0 \leq \mathrm{RP}<1$ & $\mathrm{~g} / \mathrm{g}$ produit initial \\
\hline $\mathrm{B}(t)$ & Masse de sucre rapportée à la masse de jus dans l'échantillon à l'instant $t ; 0 \leq \mathrm{B}<1$ & $g / g$ \\
\hline $\mathrm{M}_{\mathrm{p}}(t)$ & Masse de l'échantillon à l'instant $t$ & g \\
\hline$H(t)$ & Teneur en eau de l'échantillon à l'instant $t$, relativement à la masse initiale du produit & $\mathrm{g} / \mathrm{g}$ produit initial \\
\hline$t$ & Temps & $\mathrm{s}$ \\
\hline
\end{tabular}

\section{Tableau II.}

Notation et signification des paramètres utilisés pour évaluer le comportement d'échantillons de fruits immergés dans une solution de saccharose. 
- La masse de sucre rapportée à la masse de jus dans l'échantillon à l'instant t a été évaluée par le calcul :

$$
\mathrm{B}(\mathrm{t})=\mathrm{S}(\mathrm{t}) /\left[\mathrm{M}_{\mathrm{p}}(\mathrm{t}) \times \mathrm{H}(\mathrm{t})+\mathrm{S}(\mathrm{t})\right] .
$$

En effet, l'évaluation directe de cette quantité par réfractométrie pendant le procédé aurait été affectée par la modification de la texture des fruits qui deviennent de plus en plus fermes et donc de plus en plus

\section{Figure 1.}

Influence de la température sur les cinétiques de perte d'eau et de gain en soluté de rondelles de mangues soumises à un traitement par déshydratation-imprégnation par immersion dans une solution de saccharose à $50^{\circ}$ Brix.

\section{Figure 2.}

Influence de la température sur les cinétiques de perte d'eau et de gain en soluté de rondelles de mangues soumises à un traitement par déshydratation-imprégnation par immersion dans une solution de saccharose à $60^{\circ}$ Brix.

\section{Figure 3.}

Influence de la température sur les cinétiques de perte d'eau et de gain en soluté de rondelles de mangues soumises à un traitement par déshydratation-imprégnation par immersion dans une solution de saccharose à $70^{\circ} \mathrm{Brix}$.
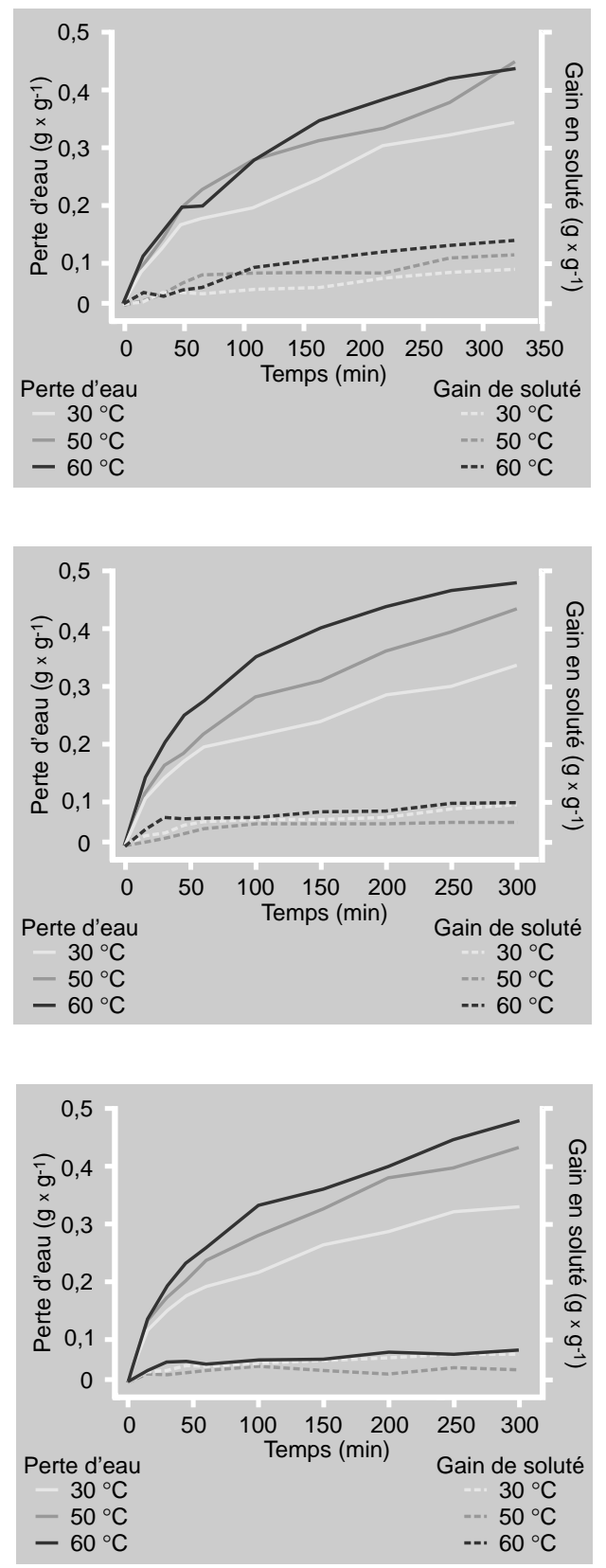

difficiles à broyer au fur et à mesure de leur évolution, ce qui entraîne une mauvaise extraction du jus.

\section{Résultats et discussions}

Les différentes températures d'incubation testées et les différentes concentrations de saccharose de la solution de déshydratation ont eu un effet sur les transferts de solutés, le degré Brix des morceaux de fruits et la diffusion de la vitamine $\mathrm{C}$.

\subsection{Effet sur les transferts de solutés}

Au cours du procédé de DII mené avec des solutions de saccharose à 50,60 ou $70^{\circ}$ Brix, la perte d'eau a augmenté en fonction de l'accroissement de la température et de la concentration de la solution (figures 1-3). Ainsi, pour qu'un échantillon perde $30 \%$ de son eau par immersion dans une solution de concentration initiale à $50^{\circ}$ Brix, il faudra en moyenne $120 \mathrm{~min}$ à $60^{\circ} \mathrm{C}$, alors qu'il faudra respectivement $150 \mathrm{~min}$ et 220 min pour perdre une même quantité d'humidité à $50{ }^{\circ} \mathrm{C}$ et $30^{\circ} \mathrm{C}$. À $60^{\circ} \mathrm{C}$, cette perte d'eau sera atteinte en 55 min et $45 \mathrm{~min}$ pour des concentrations initiales de la solution de, respectivement, $60^{\circ}$ Brix et $70^{\circ}$ Brix. Pour l'ensemble des conditions opératoires testées, les vitesses instantanées de perte d'eau ont diminué avec le temps. De semblables résultats avaient déjà été obtenus lors du traitement de pommes [12]. Pour les échantillons de mangue, les vitesses initiales de perte d'eau ont été comprises entre $6,1 \times 10^{-3}$ et $1,1 \times 10^{-2} \mathrm{~g} \times \mathrm{g}^{-1} \times \mathrm{min}^{-1}$. Ces vitesses initiales de perte d'eau sont en moyenne deux fois plus faibles que celles qui avaient été mesurées par Jiokap Nono et al. sur la pomme, à des conditions similaires de température et de concentration [12]. Cette différence pourrait être liée aux différences de texture entre ces deux fruits.

Pour l'ensemble des conditions opératoires testées, les valeurs finales de gain en soluté, comprises entre $4,2 \mathrm{~g} \times 100 \mathrm{~g}^{-1}$ et $12,0 \mathrm{~g} \times 100 \mathrm{~g}^{-1}$, ont été faibles. Aux fortes températures, le gain en soluté semble diminuer avec la concentration de la solution 
Tableau III.

Perte d'eau, gain en soluté et diminution de poids de rondelles de mangues après 300 min d'immersion dans une solution de saccharose donnée, maintenue à une température déterminée.

\begin{tabular}{|c|c|c|c|c|}
\hline $\begin{array}{l}\text { Concentration } \\
\text { de la solution de saccharose } \\
\text { ( }{ }^{\circ} \text { Brix) }\end{array}$ & $\begin{array}{l}\text { Température } \\
\text { de la solution } \\
\left({ }^{\circ} \mathrm{C}\right)\end{array}$ & $\begin{array}{l}\text { Perte } \\
\text { d'eau }^{1}\end{array}$ & $\begin{array}{l}\text { Gain } \\
\text { en soluté }{ }^{1}\end{array}$ & $\begin{array}{l}\text { Diminution } \\
\text { de poids }^{1}\end{array}$ \\
\hline \multirow[t]{3}{*}{50} & 30 & 0,340 & 0,070 & 0,270 \\
\hline & 50 & 0,420 & 0,095 & 0,325 \\
\hline & 60 & 0,446 & 0,120 & 0,326 \\
\hline \multirow[t]{3}{*}{60} & 30 & 0,390 & 0,088 & 0,302 \\
\hline & 50 & 0,510 & 0,060 & 0,450 \\
\hline & 60 & 0,570 & 0,096 & 0,474 \\
\hline \multirow[t]{3}{*}{70} & 30 & 0,440 & 0,070 & 0,370 \\
\hline & 50 & 0,590 & 0,042 & 0,548 \\
\hline & 60 & 0,650 & 0,085 & 0,565 \\
\hline
\end{tabular}

${ }^{1} \mathrm{En} \mathrm{g} \times \mathrm{g}^{-1}$ de matière fraîche.

(figures 1-3). Pour une concentration donnée, le gain en soluté maximal est obtenu avec la température testée la plus élevée $\left(60^{\circ} \mathrm{C}\right)$. Aux fortes températures avoisinant $60{ }^{\circ} \mathrm{C}$, il pourrait y avoir une dégradation des tissus des fruits, favorisant une incorporation de soluté plus importante. Une telle influence de la température sur le gain en soluté a été rapportée par plusieurs auteurs [13-15] pour lesquels les évolutions obtenues ont été variables selon la nature du produit traité et les caractéristiques de traitement appliqués.

La réduction de poids des échantillons a pu être calculée à partir de la mesure de la quantité d'eau perdue et du gain en soluté (tableau III). Pour une concentration donnée, une température supérieure à $50^{\circ} \mathrm{C}$ n'a eu qu'une faible incidence sur la diminution de poids. En revanche, pour une température donnée, l'augmentation de la concentration de la solution a entraîné une plus grande diminution de poids. Plusieurs travaux avaient déjà montré que la réduction de poids pendant le procédé de DII augmentait avec la concentration de la solution $[16,17]$.

\subsection{Effet sur le degré Brix des fruits pendant la DII}

Les courbes illustrant l'évolution, en fonction du temps et pour trois températures différentes, du degré Brix des mangues montrent que le degré Brix final des échantillons augmente avec la température et la concentration de la solution (figures 4-6). La plus forte valeur finale du degré Brix de $49,1 \mathrm{~g} \times 100 \mathrm{~g}^{-1}$ a été obtenue lorsque les rondelles étaient immergées dans une solution de $70{ }^{\circ}$ Brix placée dans un bain thermostaté de $60{ }^{\circ} \mathrm{C}$, tandis que la plus faible valeur égale à $27,1 \mathrm{~g} \times 100 \mathrm{~g}^{-1}$ a correspondu au traitement caractérisé par une solution à $50^{\circ}$ Brix placée à $30^{\circ} \mathrm{C}$.

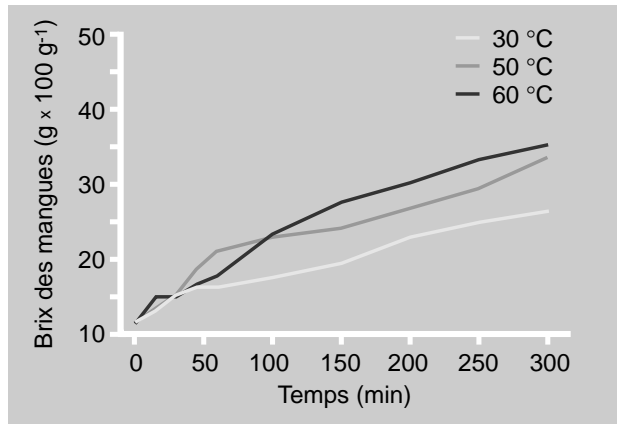

Figure 4.

Influence de la température sur les cinétiques de variation du degré Brix de rondelles de mangues soumises à un traitement par déshydratation-imprégnation par immersion dans une solution de saccharose à $50^{\circ}$ Brix. 
Figure 5.

Influence de la température sur les cinétiques de variation du degré Brix de rondelles de mangues soumises à un traitement par déshydratationimprégnation par immersion dans une solution de saccharose à $60^{\circ}$ Brix.

\section{Figure 6.}

Influence de la température sur les cinétiques de variation du degré Brix de rondelles de mangues soumises à un traitement par déshydratationimprégnation par immersion dans une solution de saccharose à $70^{\circ}$ Brix.

\section{Figure 7.}

Influence de la température sur les cinétiques de perte en vitamine $\mathrm{C}$ de rondelles de mangues soumises à un traitement par déshydratationimprégnation par immersion dans une solution de saccharose à $50^{\circ} \mathrm{Brix}$.

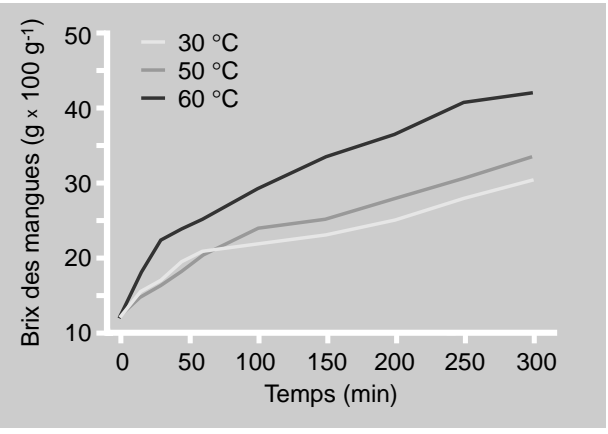

Pour les rondelles de mangues immergées dans une solution à $50^{\circ} \mathrm{Brix}$, la perte en vitamine $C$ exprimée en $\mathrm{mg} \times \mathrm{mL}^{-1}$ de solution a augmenté dans le temps et, en fin de procédé, elle a été d'autant plus importante que la température de traitement a été élevée (figure 7). Les mêmes constatations ont été faites pour les concentrations de $60{ }^{\circ}$ Brix et $70{ }^{\circ}$ Brix.

L'analyse des pertes en vitamine $C$ évaluées en fonction de la température et de la concentration de la solution de déshydratation a révèlé que, pour une température donnée, les transferts observés à $70^{\circ} \mathrm{Brix}$ étaient faibles par rapport à ceux obtenus aux concentrations inférieures de la solution d'immersion (tableau IV). Des résultats comparables avaient été obtenus par Heng et al. lors du traitement de papayes [18]. Il semble donc qu'à forte concentration, il se forme une couche de sucre à la surface des fruits, faisant office de barrière qui limiterait la diffusion ultérieure de la vitamine $\mathrm{C}$ vers la solution. Mais, à cette concentration, l'incorporation de sucre dans le fruit pourrait influencer les caractéristiques désirées du produit fini. De plus, la mise en œuvre du procédé de DII avec des solutions plus concentrées est délicate à réaliser en raison d'une part des volumes de solution mis en jeu et d'autre part de l'augmentation notable de la densité et de la viscosité de la solution. L'utilisation de conditions plus douces et limitant la diminution de poids pourrait permettre également d'obtenir des pertes relativement faibles en vitamine $C$; c'est par exemple le cas de l'emploi d'une solution de $60{ }^{\circ}$ Brix placée à $50{ }^{\circ} \mathrm{C}$ qui entraîne seulement une perte en vitamine $\mathrm{C}$ de $2,2 \mathrm{mg} \times 100 \mathrm{~g}^{-1}$ de produit initial (tableau IV). Par rapport à la teneur initiale des mangues, les pertes en vitamine $C$, mesurées à la fin du procédé de DII, sont inférieures à 12,6\%. Ces pertes se révèlent faibles par rapport à celles obtenues par Vial et al. [19], lors de la DII de kiwi. Cependant, du fait de nos conditions opératoires, les pertes en vitamine $C$ que nous avons mesurées pourraient être sous-estimées car la méthode de dosage utilisée (méthode au 2,6-dichloro-indophénol) ne quantifie que la forme active oxydable de la vitamine, laquelle serait en partie détruite pendant les traitements à 30,50 et $60^{\circ} \mathrm{C}$. 
Outre la diffusion de la vitamine $\mathrm{C}$, la mesure de la conductivité et du pH de la solution ont révélé qu'il se produisait également des pertes en sels minéraux et en acides du fruit (figure 8). Les variations de conductivité de la solution ont augmenté dans le temps et ont été d'autant plus importantes que la température de traitement a été élevée. Par ailleurs, la migration des acides des échantillons de mangue vers la solution a été plus importante au début du traitement du fait des gradients de concentration en présence, puis elle a progressivement diminué dans le temps, ce qui explique l'évolution constatée du $\mathrm{pH}$ de la solution (figure 8).

Pour limiter la diffusion des constituants nobles du fruit vers la solution d'immersion, il pourrait être conseillé de réutiliser cette même solution pour le traitement de nouveaux lots de fruits. Une étude du recyclage des solutions concentrées en DII de pommes faite par Valdez Fragoso [20] a montré que les caractéristiques de la solution (notamment le $\mathrm{pH}$, la conductivité, la teneur en sucres réducteurs, la turbidité) pouvaient être stabilisées après six recyclages. Cet auteur a également mis en évidence l'efficacité de la reconcentration puis du recyclage de la solution concentrée, par rapport au traitement classique de DII sans reconcentration.

\section{Conclusion}

Les résultats que nous avons obtenus montrent que la température et la concentration de la solution d'immersion influencent les principaux transferts d'éléments intervenant lors du procédé de DII appliqué au traitement de rondelles de mangues.

La perte d'eau et le degré Brix final des mangues ont augmenté avec la température et la concentration. Pour une température donnée, une augmentation de concentration de la solution d'immersion a favorisé la réduction de poids et diminué le gain en soluté. La perte d'eau, le gain en soluté et le degré Brix des échantillons de mangue étudiés ont été respectivement compris entre 34 et $65 \%, 4,2$ et $12 \%$ et 27,1 et $49,1 \mathrm{~g} \times 100 \mathrm{~g}^{-1}$ de jus. Au cours du procédé,

\section{Tableau IV.}

Perte en vitamine $C$ de rondelles de mangues après 300 min d'immersion dans une solution de saccharose donnée, maintenue à une température déterminée (teneur initiale en vitamine $\mathrm{C}$ des échantillons : $41,7 \mathrm{mg} \times 100 \mathrm{~g}^{-1}$ de matière fraîche).

\begin{tabular}{ccc}
$\begin{array}{l}\text { Concentration } \\
\text { de la solution } \\
\text { de saccharose }\left({ }^{\circ} \text { Brix }\right)\end{array}$ & $\begin{array}{c}\text { Température } \\
\text { de la solution } \\
\left({ }^{\circ} \mathrm{C}\right)\end{array}$ & $\begin{array}{c}\text { Perte en } \\
\text { vitamine } \mathrm{C}\end{array}$ \\
\hline 50 & 30 & 1,43 \\
& 50 & 2,45 \\
60 & 60 & 4,26 \\
60 & 30 & 0,52 \\
& 50 & 2,19 \\
70 & 60 & 5,26 \\
& 30 & 0,73 \\
& 50 & 1,08 \\
1 & 60 & 1,15
\end{tabular}

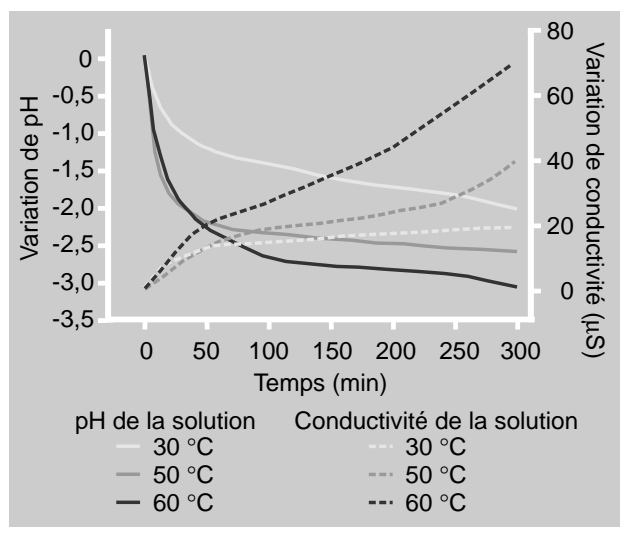

Figure 8.

Influence de la température sur la variation de $\mathrm{pH}$ et de conductivité d'une solution de saccharose à $60^{\circ} \mathrm{Brix}$ utilisée pour le traitement de rondelles de mangues par un procédé de déshydratation-imprégnation par immersion (pH initial de la solution : 7,5). la perte en vitamine $\mathrm{C}$ a été d'autant plus importante que la température a été élevée et que la concentration de la solution a été faible. Pour l'ensemble des conditions expérimentales testées, cette perte a été inférieure à $5,3 \mathrm{mg} \times 100 \mathrm{~g}^{-1}$ de produit initial.

\section{Remerciements}

Ce travail a été soutenu par l'agence universitaire de la Francophonie (Aupelf-Uref) 
dans le cadre d'un projet du fonds international de Coopération universitaire (Ficu).

\section{Références}

[1] De Laroussilhe F., Le manguier, G.P. Maisonneuve and Larose, Techniques Agricoles et Productions Tropicales, Paris, 1980.

[2] Ihekoronye A.I., Ngoddy P.O., Tropical fruits and vegetables, In: Integrated food science and technology for the tropics, (Chapter 19) Macmillan Publishers Ltd, London, UK, 1985, pp. 293-311.

[3] Woot-Tsuen Wu Leung, Food composition table for use in Africa, Food and Agriculture Organization of the United Nations, Roma, Italy, 1968.

[4] Moy J.H., Lau N.B.H., Dollar A.M., Effects of sucrose and acids on osmovac-dehydration of tropical fruits, J. Food Process. Pres. 2 (1978), 131-135.

[5] Ramamurthy M.S., Bongirwar D.R., Bandyopadhyay C., Osmotic dehydration of fruits: possible alternative for freeze drying, Indian Food Packer 32 (1978) 108-111.

[6] Uzuegbu J.O., Ukeka C., Osmotic dehydration as a method of preserving fruits to minimize ascorbic acid loss, J Food Agric. 1 (1987) 187-188.

[7] Collignan A., Raoult-Wack A.-L., Themelin A., Energy study of food processing by osmotic dehydration and air drying, Agric. Eng. J. 1 (3) (1992) 125-135.

[8] Welti J., Palou E., Lopez Malo A., Balseira A., Osmotic concentration of mango slices, Dry Technol. 13 (1995) 405-416.

[9] Anonymous, Official methods of analysis (13 $13^{\text {th }}$ edition), Association of Official Analytical Chemists (AOAC), Washington, DC, USA, 1980.

[10] Audigie C., Figarella J., Zonszain F., Manipulation d'analyse biochimique, Doin (Éd.), Paris, France, 1980, 274 p.

[11] Jiokap Nono Y., Nuadje G.B., Raoult-WackA.-L., Giroux F., Comportement de certains fruits tropicaux traités par déshydratation-imprégnation par immersion dans une solution de saccharose, Fruits 56 (2) 75-83.

[12] Jiokap Nono Y., Giroux F., Cuq B., RaoultWack A.-L., Étude des paramètres de contrôle et de commande du procédé de déshydratation-imprégnation par immersion sur système probatoire automatisé : application au traitement des pommes Golden, J. Food Eng. 2001 (sous presse).

[13] Lenart A., Flink J.M., Osmotic concentration of potato. I. Criteria for the end point of osmotic process, J. Food Technol. 19 (1984) 45-63.

[14] Vargas P., Étude de la déshydratation osmotique et du semi-confisage couplé au séchage de banane. Possibilité d'application au Costa Rica, Mémoire de mastère spécialisé en Technologie agroalimentaire pour les régions chaudes, Ensia-Siarc, Montpellier, France, 1989.

[15] Raoult Wack A.-L., Les procédés de DII dans les solutions concentrées : étude expérimentale et modélisation des transferts d'eau et de soluté sur gel modèle, Université de Montpellier II, Thèse, France 1991.

[16] Hawkes J., Flink J.M., Osmotic concentration of fruits slices prior to freeze dehydration, J. Food Process. Pres. 2 (1978) 265-284.

[17] Conway J., Castaigne F., Picard G., Vova X., Mass transfer consideration in the osmotic dehydration of apples, Can. Inst. Food. Sci. Technol. J. 16 (1983) 25-29.

[18] Heng K., Guilbert S., Cuq J.L., Osmotic dehydration of papaya. Influence of process variables on the product quality, Sci. Aliment 10 (1990) 831-848.

[19] Vial C., Guilbert S., Cuq J.L., Osmotic dehydration of kiwi fruits: influence of process variables on the color and ascorbic acid content, Sci. Aliment 11 (1991) 63-84.

[20] Valdez Fragoso A., Étude du recyclage des solutions concentrées en déshydratationimprégnation par immersion. Application au cas du traitement de pommes dans une solution de saccharose, Ensia, Thèse, Massy, France, 1998. 
Deshidratación-impregnación por inmersión (DII) de rodajas de mango (Mangifera indica): influencia de la temperatura y de la concentración de la solución en las cinéticas de ciertos elementos constitutivos del fruto.

Resumen - Introducción. Las transferencias de materia que intervienen durante el procedimiento de DII influyen en la composición final del producto. Dichas transferencias dependen esencialmente de la forma y tamaño de los productos inmersos, así como de la temperatura, concentración y naturaleza de la solución de inmersión. Para lograr un buen dominio de estos parámetros y obtener, tras tratamiento, un producto de calidad, se siguieron las cinéticas de ciertos elementos constitutivos de los mangos (Mangifera indica) durante un tratamiento mediante DII. Material y métodos. Se introdujeron trozos de mango, de una variedad camerunesa local, de $9 \mathrm{~mm}$ de grosor y $31 \mathrm{~mm}$ de diámetro en una solución de sacarosa a 50, 60 ó $70{ }^{\circ}$ Brix, mantenida a una temperatura estabilizada a 30, 50 ó $60^{\circ} \mathrm{C}$. En las muestras, se midió la pérdida de agua, reducción de peso, ganancia en solutos y grado Brix mientras que, en la solución, se midió la concentración en vitamina $\mathrm{C}, \mathrm{pH}$ y conductividad. Resultados y discusión. La pérdida de agua y el grado Brix final de los productos aumentaron con la temperatura y la concentración de la solución. Por encima de $50{ }^{\circ} \mathrm{C}$, el aumento de temperatura no ha influido significativamente en la reducción del peso que, a una temperatura dada, ha aumentado con la concentración de la solución de inmersión. La ganancia en solutos disminuyó con el aumento de la concentración. Por último, en las condiciones de operaciones probadas, las pérdidas de vitamina $\mathrm{C}$ aumentaron con la temperatura y disminuyeron con el aumento de la concentración de sacarosa.

Camerún / Mangífera indica / conservación (almacenamiento) / inmersión / temperatura / sucrosa / concentración / deshidratación osmótica / difusión / acido ascorbico / soluto / contenido de humedad / contenido de carbohidratos / pH / conductividad eléctrica 